Supporting Information

\title{
Polar Molecular Modification onto BiOBr to Regulate Molecular Oxygen Activation
}

Xinhua Song +, Jiajia Wang ,, Ruoqian Zhang ',Yuanyuan Liu * *, Guangwei Yu ,

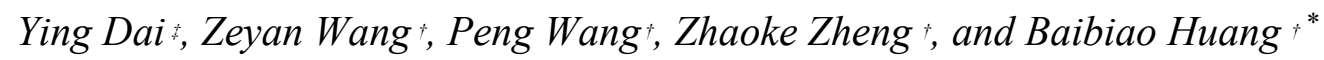

$†$ State key of Crystal Materials, Shandong University, Jinan 250100, P. R. China

† School of Physics, Shandong University, Jinan 250100, P. R. China

*E-mail: yyliu@sdu.edu.cn; bbhuang@sdu.edu.cn. 


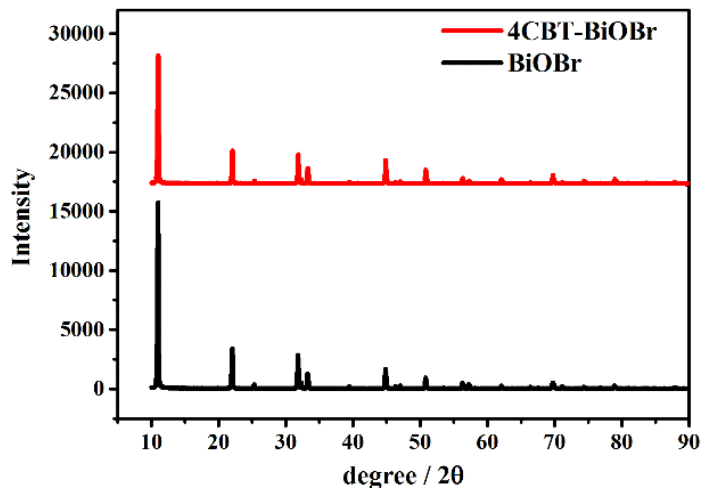

Figure. S1. X-ray diffraction (XRD) pattern of the $\mathrm{BiOBr}$ and $4 \mathrm{CBT}-\mathrm{BiOBr}$

$\mathrm{BiOBr}$ and 4CBT-BiOBr sample are synthesized through a modified hydrothermal method according to previous report ${ }^{1-2}$. As shown in Figure S1, X-ray diffraction (XRD) pattern of the sample can be assigned to typical BiOBr structure (JCPDS card No. 73-2061), suggesting its high purity with orientation along the [001] direction. The XRD spectra of 4CBT-BiOBr remains unchanged from $\mathrm{BiOBr}$. The results show that the 4CBT small molecule does not change the bulk structure of $\mathrm{BiOBr}$ in good agreement with those reported in the literature. 


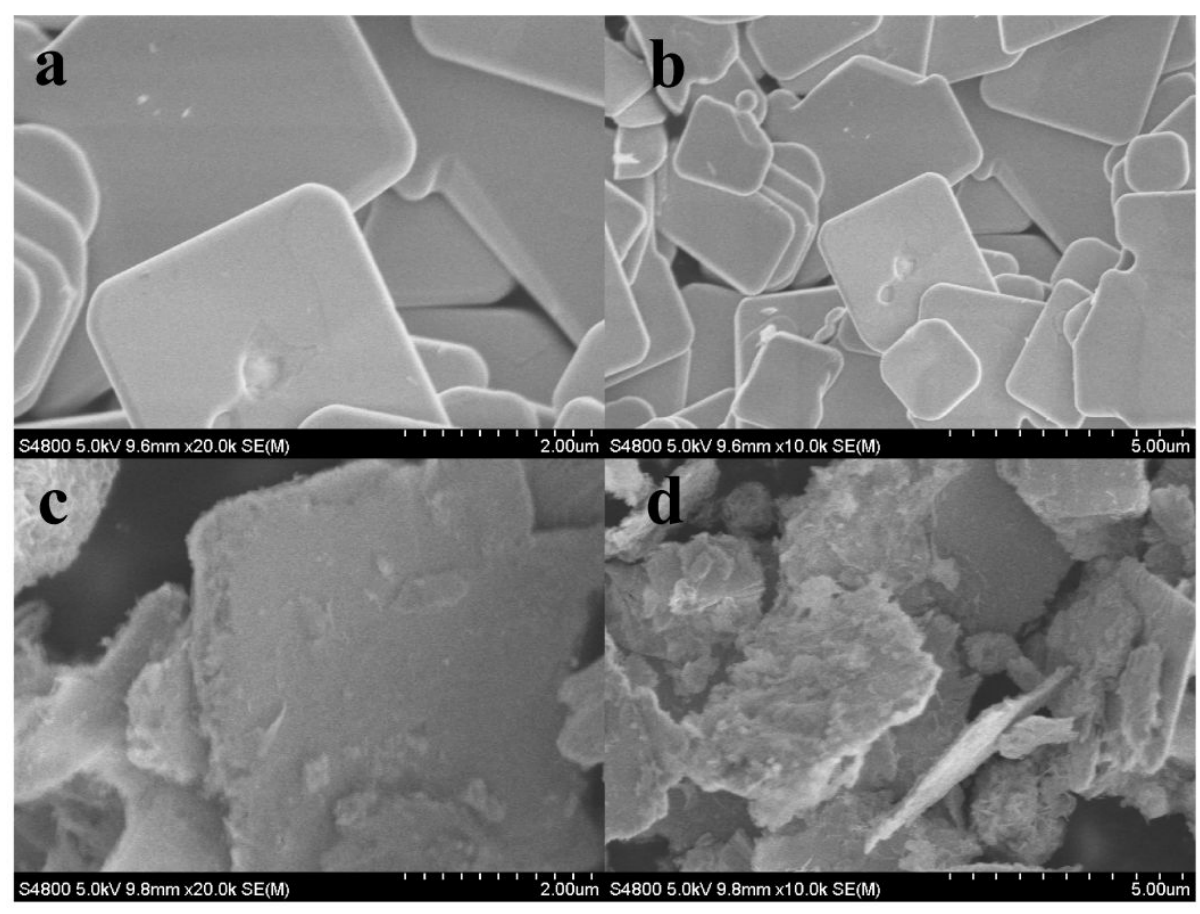

Figure S2. SEM images of (a b) BiOBr and (b d) 4CBT-BiOBr.

\section{Supporting References}

(1) Xu, B.; An, Y.; Liu, Y.; Huang, B.; Qin, X.; Zhang, X.; Dai, Y.; Whangbo, M. H. An efficient visible-light photocatalyst made from a nonpolar layered semiconductor by grafting electron-withdrawing organic molecules to its surface. Chem Commun (Camb) 2016, 52, 13507-13510.

(2) Xu, B.; An, Y.; Liu, Y.; Qin, X.; Zhang, X.; Dai, Y.; Wang, Z.; Wang, P.; Whangbo, M.-H.; Huang, B. Enhancing the photocatalytic activity of $\mathrm{BiOX}(\mathrm{X}=\mathrm{Cl}$, $\mathrm{Br}$, and $\mathrm{I}),(\mathrm{BiO})_{2} \mathrm{CO}_{3}$ and $\mathrm{Bi}_{2} \mathrm{O}_{3}$ by modifying their surfaces with polar organic anions, 4-substituted thiophenolates. Journal of Materials Chemistry A 2017, 5, 14406-14414. 